\title{
Squark/gluino in leptonic channels with ATLAS
}

\author{
Martina JAVURKOVA* \\ On behalf of the ATLAS collaboration \\ Albert-Ludwigs-Universitaet Freiburg (DE) \\ E-mail: martina.javurkova@cern.ch
}

Weak-scale supersymmetry is one of the most motivated theories for physics beyond the Standard Model. This proceeding summarizes some of the recent ATLAS results on inclusive searches for the supersymmetric partners of quarks and gluons in proton-proton collisions at a centre-of-mass energy of $13 \mathrm{TeV}$ and with an integrated luminosity of $3.2 \mathrm{fb}^{-1}$. These analyses target strong production of supersymmetic particles using events with jets, missing transverse momentum and leptons. No significant excess above Standard Model expectations is observed and exclusion limits have been set on squark and gluino masses in various scenarios. The limits obtained extend the ones obtained during Run- 1 of the LHC.

Fourth Annual Large Hadron Collider Physics

13-18 June 2016

Lund, Sweden

${ }^{*}$ Speaker. 


\section{Introduction}

Supersymmetry (SUSY) [1] is an extension to the Standard Model (SM) which provides a solution to the hierarchy problem by predicting the existence of a bosonic supersymmetric partner for each known fermion and vice-versa. A new additive quantum number, $R$-parity, has been introduced to prevent rapid proton decay resulting in the pair production of SUSY particles and leaving the lightest of them stable. In a large variety of models, the lightest supersymmetric particle (LSP) is either the lightest neutralino $\tilde{\chi}_{1}^{0}$ or the gravitino $\tilde{G}$, providing a dark matter particle candidate.

The analyses presented in this proceeding focus on the searches for the first and second generation of scalar quarks (squarks) and the fermionic partner of the gluon (gluino). If the squark/gluino can be directly produced in $p p$ collisions, then their pair production dominates at the Large Hadron Collider (LHC). They decay in a cascade up to the LSP leading to a final state with many high- $p_{\mathrm{T}}$ jets, large missing transverse momentum and possibly leptons. The searches discussed here target only the leptonic decay channels. The results are interpreted in the context of simplified scenarios where branching fractions are set to $100 \%$. Examples of the topologies considered for gluinomediated production with the direct decay $\left(\tilde{g} \rightarrow t t \tilde{\chi}_{1}^{0}\right)$ and with up to two extra steps in the decay chain $\left(\tilde{g} \rightarrow q q \tilde{\chi}_{1}^{ \pm} \rightarrow q q \nu_{\tau} \tilde{\tau} \rightarrow q q \nu_{\tau} \tau \tilde{\chi}_{1}^{0}\right)$ are shown in Figure 1. A gauge-mediated SUSY-breaking (GMSB) model with more complex decay chains is investigated as well.

Early Run-2 searches have been performed with $3.2 \mathrm{fb}^{-1}$ of data collected with the ATLAS detector at a centre-of-mass energy of $13 \mathrm{TeV}$. They all followed simplified search strategies as compared to the corresponding Run-1 analyses.
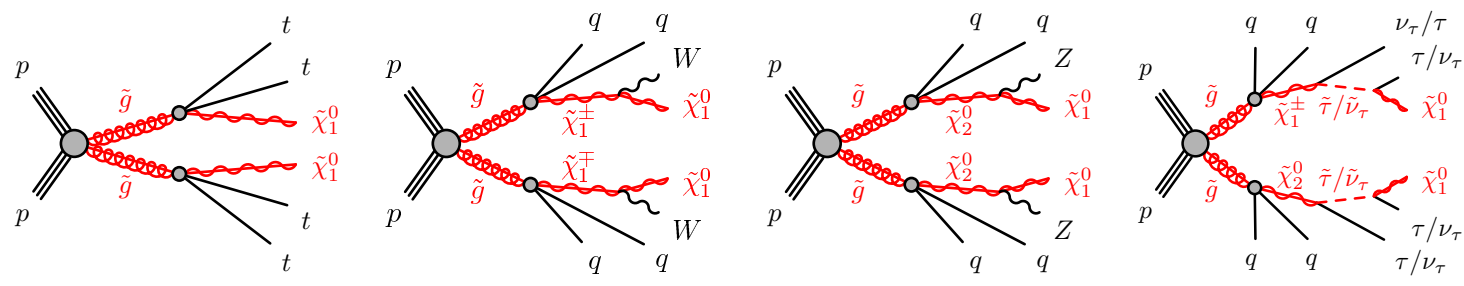

Figure 1: Feynman diagrams for gluino pair production and possible decays $[2,3,4,5]$.

\section{Search strategy}

The background estimation methods as well as the interpretation of the results are similar among the various SUSY analyses. Given that SUSY searches are typically performed in corners of phase space with large missing transverse momentum and/or high transverse momentum objects, the SM background needs to be precisely known here. The main irreducible backgrounds from the SM processes $(W+$ jets, $Z+$ jets, top production, etc.) are estimated via partially data-driven techniques using control regions. These are enriched in the background process of interest and designed to be kinematically close but orthogonal to the regions with enhanced signal (signal regions). The Monte Carlo (MC) simulations are then normalized to data and extrapolated to the signal regions. The rarer processes ( $t \bar{t} V$, dibosons, etc.) are directly taken from simulated samples using the production cross-section predicted by the theory. The reducible backgrounds (misidentified leptons, 
etc.) are estimated using fully data-driven methods. All background yields are tested with data in validation regions chosen to be closer to the signal regions.

The upper limits are set at the 95\% confidence level using the HistFitter framework [6]. The one-sided profile-likelihood-ratio test statistic is used to assess the compatibility of the observed data with the expected background yields in the signal regions.

\section{Search in final states with 1 lepton}

The experimental signature characterised by one isolated charged electron or muon, several jets, and missing transverse momentum $E_{\mathrm{T}}^{\text {miss }}$ allows to target SUSY models with various mass differences between the predicted particles using two complementary sets of signal regions. The first set requires a low- $p_{\mathrm{T}}$ lepton and the second a high- $p_{\mathrm{T}}$ lepton. The signal model considered in this search [2] is a simplified model where pair-produced gluinos decay via the lightest chargino to the lightest neutralino. The SM processes with similar kinematics are suppressed by stringent requirements on the transverse mass $m_{\mathrm{T}}$ and the inclusive effective mass $m_{\mathrm{eff}}^{\text {inc }}$ which is the scalar sum of the $p_{\mathrm{T}}$ of the signal lepton, jets and the $E_{\mathrm{T}}^{\mathrm{miss}}$. Other variables providing good discrimination against the background are the transverse momentum scalar sum $H_{\mathrm{T}}$, the ratio $E_{\mathrm{T}}^{\mathrm{miss}} / m_{\mathrm{eff}}^{\mathrm{inc}}$ and the jet aplanarity [7]. The dominant background processes after the full event selection are $t \bar{t}$ and $W+$ jets. They are normalised to the number of data events observed in dedicated control regions. Since no significant excess above the SM expectation is observed, the exclusion limits are set for scenarios with large and small mass differences between the gluino and the neutralino (Figure 2).
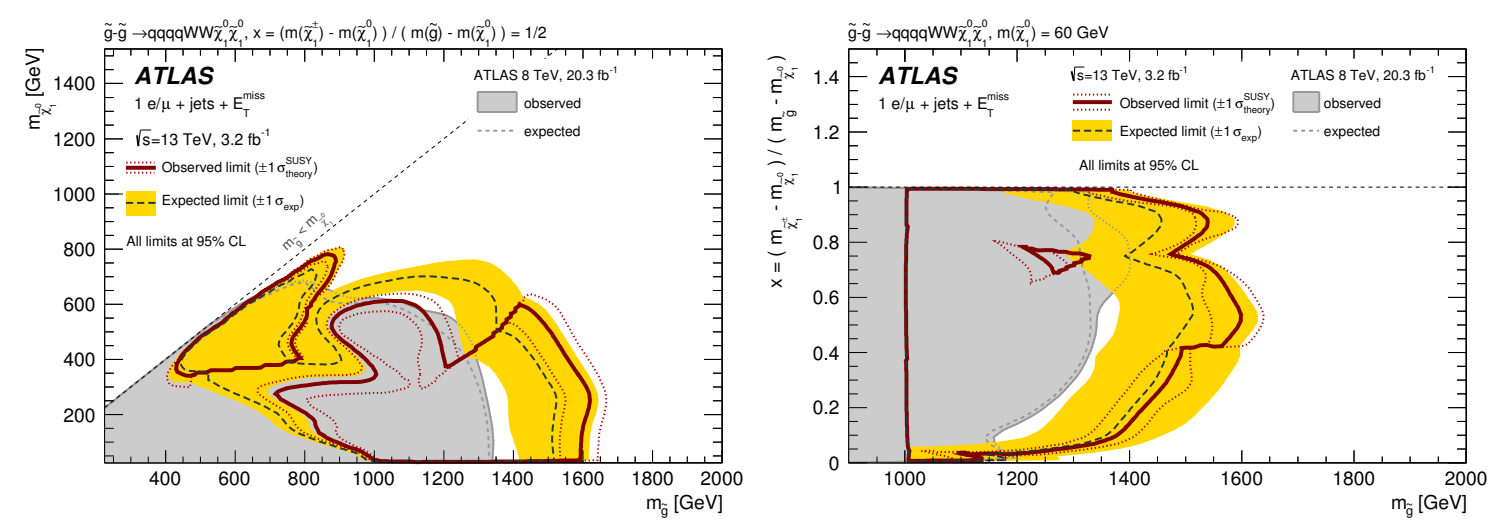

Figure 2: Observed and expected exclusion limits at 95\% confidence level for the simplified models of the gluino pair production in the context of two scenarios [2]. The limits are presented in the $\left(m_{\tilde{g}}, m_{\tilde{\chi}_{1}^{0}}\right)$ mass plane (left) for the scenario where the mass of the chargino is fixed to $x=\left(m_{\tilde{\chi}_{1}^{ \pm}}-m_{\tilde{\chi}_{1}^{0}}\right) /\left(m_{\tilde{g}}-m_{\tilde{\chi}_{1}^{0}}\right)=1 / 2$ and in the $\left(m_{\tilde{g}}, x\right)$ mass plane (right) for the $m_{\tilde{\chi}_{1}^{0}}=60 \mathrm{GeV}$ scenarios. The exclusion limits by previous ATLAS analyses [8] are shown as the grey area.

\section{Search in final states with 2 leptons}

A topology with large $E_{\mathrm{T}}^{\text {miss }}$, jets and two opposite-sign leptons produced from a $Z$ boson decay is sensitive to supersymmetric events where $Z$ boson itself is produced in a cascade decay 
of a gluino. An excess with a significance of 3 standard deviations was observed in the ATLAS analysis performed with $\sqrt{s}=8 \mathrm{TeV}$ collision data using this final state [9]. Therefore, the analysis was repeated with the first $\sqrt{s}=13 \mathrm{TeV}$ data and identical kinematic selections [3]. The selected events are required to be within the $Z$-mass window of $81<m_{\ell \ell}<101 \mathrm{GeV}$, contain at least two jets and have high values of $E_{\mathrm{T}}^{\text {miss }}$ and $H_{\mathrm{T}}$. The background yields from the "flavour-symmetric" processes $(t \bar{t}, W W$ and $W t)$ and $Z / \gamma^{*}+$ jets are estimated using data-driven methods and carefully validated. More events are observed than predicted, corresponding to a significance of $2.2 \sigma$. The expected and observed exclusion contours are shown in Figure 3.

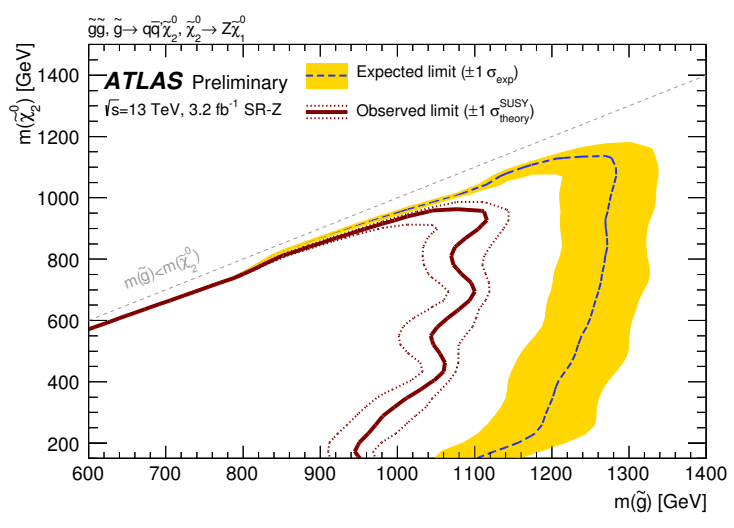

Figure 3: Observed and expected exclusion limits at $95 \%$ confidence level for the simplified model of the gluino pair production with onestep decay $\tilde{g} \rightarrow q \bar{q}^{\prime} \tilde{\chi}_{2}^{0} \rightarrow q \bar{q}^{\prime} Z \tilde{\chi}_{1}^{0}$ and with the LSP mass set to $1 \mathrm{GeV}[3]$.

\section{Search in final states with 2 same-sign or 3 leptons}

The analysis searching for SUSY in the events with two same-sign or three leptons, jets and $E_{\mathrm{T}}^{\text {miss }}$ [4] benefits from low SM backgrounds and thus looser kinematic requirements can be applied. The search is sensitive to many theories beyond the SM, allowing interpretation of the results in the context of four simplified SUSY models. Four signal regions are designed to maximize the sensitivity for each scenario. They are characterized by the lepton and $b$-jet multiplicity. The background events containing at least one lepton which originates from hadron decays or electrons with mis-measured charge are estimated with data-driven methods, whilst events with two same-sign prompt leptons or at least three prompt leptons coming mainly from $t \bar{t} V$ and diboson processes are estimated with MC simulations. No excess is found in neither of the signal regions and constraints are set on the corresponding SUSY parameter spaces. Figure 4 shows the exclusion limits in the mass plane of the lightest neutralino and gluino for two different gluino decay chains. They are compared to the limits obtained by the combination of $8 \mathrm{TeV}$ ATLAS SUSY searches. The sensitivity exceeds that of the Run-1 data in some parts of the phase space.

\section{Search in final states with $\tau$ leptons}

The search for squarks and gluinos in events with jets, at least one hadronically decaying tau lepton and large missing transverse momentum [5] considers two SUSY scenarios: a simplified model of gluino pair production and a gauge-mediated SUSY-breaking (GMSB) model. Two 

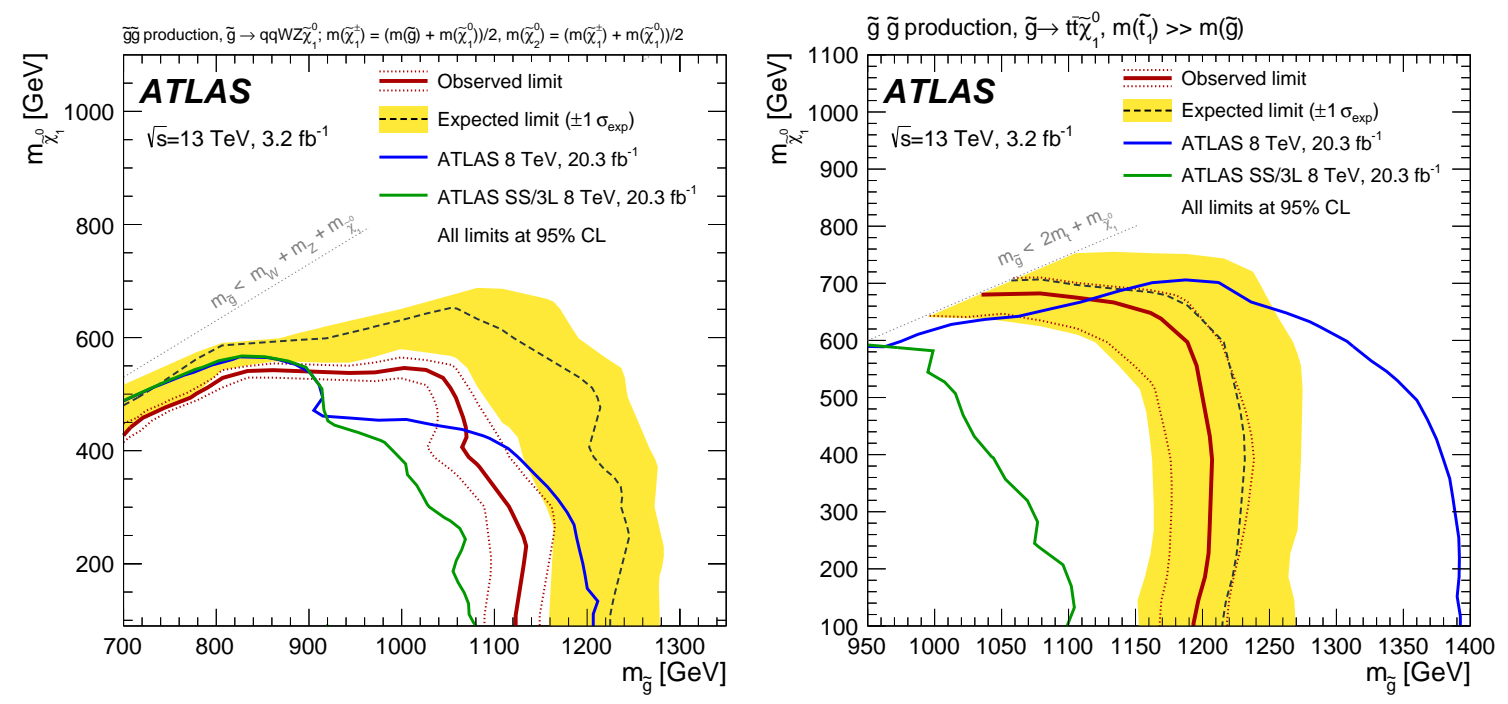

Figure 4: Observed and expected exclusion limits at 95\% confidence level for the simplified models of the gluino pair production with exclusive decay modes $\tilde{g} \rightarrow q \bar{q}^{\prime} W Z \tilde{\chi}_{1}^{0}$ with the intermediate particle masses set to $m_{\tilde{\chi}_{1}^{ \pm}}=\left(m_{\tilde{g}}+m_{\tilde{\chi}_{1}^{0}}\right) / 2$ and $m_{\tilde{\chi}_{2}^{0}}=\left(m_{\tilde{\chi}_{1}^{ \pm}}+m_{\tilde{\chi}_{1}^{0}}\right) / 2$ (left), and $\tilde{g} \rightarrow t \bar{t} \tilde{\chi}_{1}^{0}$ (right) [4]. The results are compared with the observed limits obtained by previous ATLAS searches [10, 8, 11].

exclusive channels, with one tau lepton $(1 \tau)$ and at least two tau leptons $(2 \tau)$, are studied and optimised separately. In each topology, several signal regions are defined for various mass-splitting scenarios. The main discriminant variables are the transverse mass and the stransverse mass [12]. The normalisation of the dominant backgrounds ( $t \bar{t}$ and $W / Z+$ jets) is obtained via a simultaneous likelihood fit to data in the dedicated control regions. For the simplified model, the exclusion contours are derived in the $\left(m_{\tilde{g}}, m_{\tilde{\chi}_{1}^{0}}\right)$ plane and are shown for both channels individually, as well as their combination in Figure 5 (left). The GMSB model is probed as a function of $\Lambda$ and $\tan \beta$, while the other parameters are fixed. The results based on the $2 \tau$ channel are shown in Figure 5 (right).
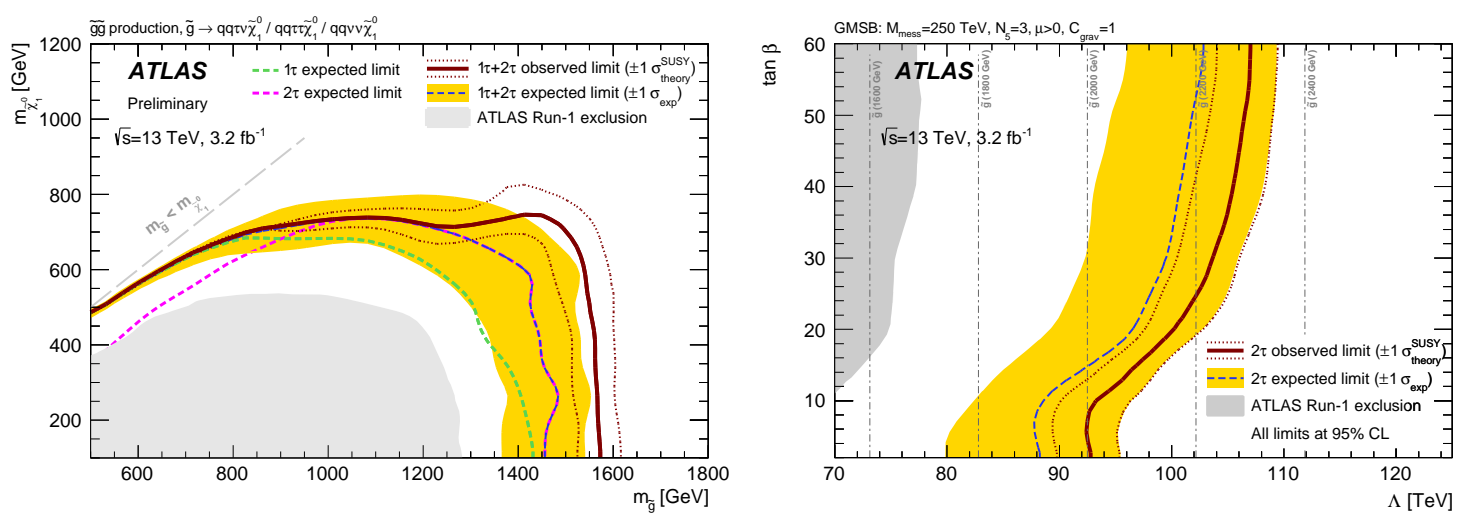

Figure 5: Observed and expected exclusion limits at 95\% confidence level for the simplified model of gluino pair production with $m_{\tilde{\chi}_{1}^{ \pm}}=m_{\tilde{\chi}_{2}^{0}}=\left(m_{\tilde{g}}+m_{\tilde{\chi}_{1}^{0}}\right) / 2$ and $m_{\tilde{\tau}}=m_{\tilde{v}_{\tau}}=\left(m_{\tilde{\chi}_{1}^{ \pm}}+m_{\tilde{\chi}_{1}^{0}}\right) / 2$ (left), and for the gaugemediated supersymmetry-breaking model (right) [5]. The previous ATLAS results [8, 13] obtained with $20.3 \mathrm{fb}^{-1}$ of $8 \mathrm{TeV}$ data are shown as the grey filled area. 


\section{Conclusion}

The ATLAS collaboration has performed many searches for squarks and gluinos using the first $3.2 \mathrm{fb}^{-1}$ of collected $p p$ data at $\sqrt{s}=13 \mathrm{TeV}$. None of the analyses have shown a statistical significant deviation from the Standard Model predictions. Although a simple search strategy has been employed, the exclusion limits significantly extended the corresponding Run-1 results.

\section{References}

[1] S. P. Martin, Adv. Ser. Direct. High Energy Phys. 21 (2010), arXiv:hep-ph/9709356

[2] ATLAS Collaboration, arXiv:1605.04285

[3] ATLAS Collaboration, ATLAS-CONF-2015-082

[4] ATLAS Collaboration, Eur. Phys. J. C76 (2016) 259, arXiv:1602.09058

[5] ATLAS Collaboration, arXiv:1607.05979

[6] M. Baak, G. J. Besjes, D. Côte, A. Koutsman, J. Lorenz and D. Short, Eur. Phys. J. C75 (2015) 153, arXiv: 1410.1280

[7] C. Chen, Phys. Rev. D 85 (2012) 034007, arXiv:1112.2567

[8] ATLAS Collaboration, JHEP 1510 (2015) 054, arXiv:1507.05525

[9] ATLAS Collaboration, Eur. Phys. J. C75 (2015) 318, arXiv:1503.03290

[10] ATLAS Collaboration, JHEP 06 (2014) 035, arXiv:1404.2500

[11] ATLAS Collaboration, Eur. Phys. J. C75 (2015) 510, arXiv:1506.08616

[12] C. G. Lester and D. J. Summers, Phys. Lett. B463 (1999) 99, arXiv:hep-ph/9906349

[13] ATLAS Collaboration, JHEP 1409 (2014) 103, arXiv:1407.0603 